\title{
Nonlinear Analysis of a Maglev System with Time-delayed Feedback Control
}

\author{
Lingling Zhang, ${ }^{\mathrm{a}, \mathrm{b}}$, Sue Ann Campbell ${ }^{\mathrm{b}, *}$, Lihong Huang ${ }^{\mathrm{a}}$ \\ ${ }^{a}$ Hunan Women's University, Changsha, Hunan, 410002, P. R. China \\ ${ }^{b}$ Department of Applied Mathematics, University of Waterloo, Waterloo, ON, N21 3 G1 Canada
}

\begin{abstract}
This paper undertakes a nonlinear analysis of a model for a maglev system with time-delayed feedback. Using linear analysis, we determine constraints on the feedback control gains and the time delay which ensure stability of the maglev system. We then show that a Hopf bifurcation occurs at the linear stability boundary. To gain insight into the periodic motion which arises from the Hopf bifurcation, we use the method of multiple scales on the nonlinear model. This analysis shows that for practical operating ranges, the maglev system undergos both subcritical and supercritical bifurcations, and which give rise to unstable and stable limit cycles respectively. Numerical simulations confirm the theoretical results and indicate that unstable limit cycles may coexist with the stable equilibrium state. This means that large enough perturbations may cause instability in the system even if the feedback gains are such that the linear theory predicts that the equilibrium state is stable.

Keywords: Maglev system, Time delayed feedback, Hopf bifurcation, Method of multiple scales
\end{abstract}

2010 MSC: 70K50, 93D15, 34K20

\section{Introduction}

The maglev train is a novel type of rail vehicle that has many advantages such as high speed, comfort, low environmental pollution, and low maintenance cost $[1,2,3]$. However, the maglev system is a complicated system with machinery, controllers and electromagnetic

\footnotetext{
${ }^{*}$ Corresponding author. Tel.: +1 5198884567; fax: +1 5197464319.
}

E-mail address: sacampbell@uwaterloo.ca. 
elements integrated together. The electromagnetic suspension provides non-contacting support by means of electromagnets in conjunction with a position regulator which uses position (that is air-gap), velocity and acceleration feedback. During experiments, it is observed that time delay is unavoidable in the control and execution processes of the maglev system. For example, time delays occur in calculating the control variables, in signal processing and in digital filtering. Further, it is well known that time delay can cause instability of a closed loop system. Thus it is very necessary to investigate the dynamic behaviors of the nonlinear maglev system with time-delay.

Time-delayed systems, which have been studied for various applications and control systems, may admit rich dynamics, including bifurcations and chaotic motions. M. Attilio $[4,5,6]$ has pointed out that time-delay can limit sympathetic vibrations. Stone and Campbell [7] used center manifold theory to analyze the Hopf bifurcation of a variable-speed drill, and found that different drill speeds coincide with several regions of local stability. G. Stépán [8] analyzed the delayed positoning of a single-degree-of-freedom robot arm and showed that the system exists a codimension two Hopf bifurcation. G. Orosz and G. Stépán [9] investigated the bifurcation phenomenon in traffic system. N.A. Nayfeh [10] concluded that the controller of a container crane undergoes a supercritical bifurcation for practical operating ranges.

Several valuable achievements have been made in the research of nonlinear dynamic and bifurcation phenomenon for the maglev system. Using various techniques (centre manifold reduction, pseudo-oscillator analysis), previous work [11, 12, 13] studied the stability and Hopf bifurcation of the single-degree-of-freedom suspension system with time delay, and found that the dynamic behavior can be changed by adjusting time delay. But the time delay of state feedback signals for the maglev system is not adjustable in practice. So the focus of our work is on how the control gains affect the stability and Hopf bifurcation of the maglev time-delay feedback system.

In this paper, we present a linear stability analysis of time-delay feedback controller to determine constraints on the feedback gains and time delay which ensure the system is stabilized. The linear analysis shows that the maglev system undergoes a Hopf bifurcation for certain parameter values. Choosing the velocity feedback gain as the bifurcation parameter, 
we obtain the normal form of the Hopf bifurcation using the method of multiple scales. With the normal form we obtain, the type of bifurcation of the maglev system and the stability of the resulting limit cycle can be determined.

Since A.H. Nayfeh $[14,15]$ first presented the method of multiple scales, it has been successfully used in practical design engineering $[16,17,18]$. The method of multiple scales not only reveals the complex dynamic of nonlinear systems, but also provides useful approximate formulas for the periodic solution. In many cases it is easier to use than the centre manifold approach [19].

This paper is organized as follows. In Section 2, we give the linear analysis of the maglev time-delay feedback system and show that a Hopf bifurcation occurs at the linear stability boundary. In Section 3 we derive the normal form and determine the criticality of the Hopf bifurcation using the method of multiple scales. In Section 4 we show numerical studies which verify the theoretical analysis. Finally, in Section 5, we draw conclusions about our work and highlight the implications for the maglev system.

\section{Linear stability analysis and existence of Hopf bifurcation}

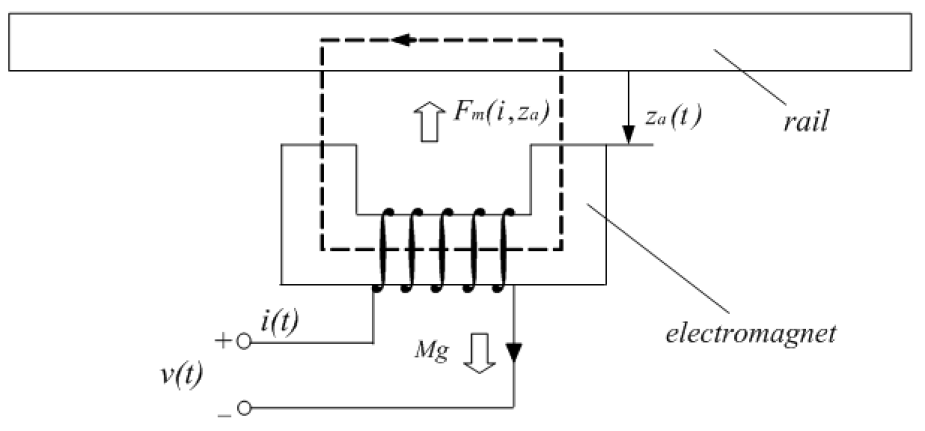

Figure 1: Structure of the maglev system.

The maglev model we study is similar to that presented in $[11,12,13,20]$. Following the approach of $[11,12,13]$, we make the simplification that the deformation of the track is zero. In this case, the schematic diagram of a single-degree-of-freedom suspension system with a controlled DC electromagnet is as shown in Fig. 1. In the diagram, $M g$ and $F_{m}$ represent 
the weight and the electromagnetic force of the electromagnet respectively, and $z_{a}$ denotes the vertical displacement of the electromagnet. The variables $i(t)$ and $v(t)$ are the current and voltage of the electromagnet winding.

Using the notation of Fig. 1, the dynamical and electromagnetic equations of the system are given as $[11,12,13,20]$

$$
\begin{aligned}
v & =r i+A_{1} \frac{\dot{i} z_{a}-i \dot{z}_{a}}{2 z_{a}^{2}}, \\
M \ddot{z}_{a} & =M g-\frac{A_{1} i^{2}}{4 z_{a}^{2}}
\end{aligned}
$$

where $A_{1}=N^{2} \mu_{0} S_{0}, \mu_{0}$ is the magnetic permeability in vacuum, $N$ is the number of turns of coil, $S_{0}$ is the pole area and $r$ is the resistance of the electromagnet. More details on the physical principles underlying this model can be found in [20, 21].

From (2), $i=2 z_{a} \sqrt{\frac{M\left(g-\ddot{z}_{a}\right)}{A_{1}}}$. Substituting this into (1) gives:

$$
\dddot{z}_{a}=\frac{4 r}{A_{1}} z_{a}\left(g-\ddot{z}_{a}\right)-2 \sqrt{\frac{\left(g-\ddot{z}_{a}\right)}{M A_{1}}} v .
$$

Typically the feedback control is applied to the port voltage of the electromagnet. Thus, taking $z_{a}, \dot{z}_{a}$ and $\ddot{z}_{a}$ as the feedback state variables, we have:

$$
v=k_{p}\left(z_{a \tau}-z_{e}\right)+k_{d} \dot{z}_{a \tau}+k_{a} \ddot{z}_{a \tau}+v_{e c}
$$

where $k_{p}, k_{d}$ and $k_{a}$ are, respectively, position, velocity and acceleration feedback control gains and $v_{e c}$ is the voltage in the static (equilibrium) state. Note that $k_{p}$ influences the steady-state error and hence the stiffness, $k_{d}$ controls the suspension damping and $k_{a}$ the overall stability margin. Some previous work $[11,12]$ assumed that the time delay occurs only in one or two of the feedback control variables, however, we make the more reasonable assumption, as in [13], that all the feedback control variables have a time delay. We use $z_{a \tau}=z_{a}(t-\tau), \dot{z}_{a \tau}$ and $\ddot{z}_{a \tau}$ to denote, respectively, the position, velocity and acceleration feedback control signals with time delay. The linear stability analysis of the equilibrium point of (3)-(4) has been considered in [13], with an emphasis on stability changes as the delay, $\tau$, is varied. Here we will consider how variation of the feedback gains as well as the delay affect the stability. For clarity, we repeat some of the linear analysis presented in [13]. 
The values of the state variables at the equilibrium point are

$$
z_{a}=z_{e}, \dot{z}_{a}=0, \ddot{z}_{a}=0
$$

where $z_{e}$ is the desired gap. Note that this gives the value of the voltage at equilibrium as $v_{e c}=2 r z_{e} \sqrt{\frac{M g}{A_{1}}}$. Setting $z=z_{a}-z_{e}$, i.e., moving the equilibrium point to the origin, and using expression (4) for the feedback, (3) becomes:

$$
\dddot{z}-A_{2}\left(z+z_{e}\right)(g-\ddot{z})=-A_{3} \sqrt{g-\ddot{z}}\left[k_{p} z_{\tau}+k_{d} \dot{z}_{\tau}+k_{a} \ddot{z}_{\tau}+v_{e c}\right],
$$

where $A_{2}=\frac{4 r}{A_{1}}, A_{3}=\frac{2}{\sqrt{M A_{1}}}$.

Note that our model breaks down when the electromagnet comes into contact with the track. This corresponds to $z=-z_{e}$, i.e., $z_{a}=0$ in the original variables.

Expanding (5) in a Taylor series in $\left(z, \dot{z}, \ddot{z}, z_{\tau}, \dot{z}_{\tau}, \ddot{z}_{\tau}\right)$, and retaining up to cubic terms, we obtain

$$
\dddot{z}=-\frac{1}{2} A_{2} z_{e} \ddot{z}-\sqrt{g} A_{3} k_{a} \ddot{z}_{\tau}-\sqrt{g} A_{3} k_{d} \dot{z}_{\tau}-\sqrt{g} A_{3} k_{p} z_{\tau}+A_{2} g z+f\left(z, \dot{z}, \ddot{z}, z_{\tau}, \dot{z}_{\tau}, \ddot{z}_{\tau}\right),
$$

where

$$
\begin{aligned}
f= & \frac{A_{3} k_{a}}{2 \sqrt{g}} \ddot{z}_{\tau}+\frac{z_{e} A_{2}}{8 g} \ddot{z}^{2}+\frac{A_{3} k_{d}}{2 \sqrt{g}} \dot{z}_{\tau} \ddot{z}+\frac{A_{3} k_{p}}{2 \sqrt{g}} \ddot{z} z_{\tau}-A_{2} z \ddot{z}+\frac{A_{3} k_{a}}{8 \sqrt{g^{3}}} \ddot{z}_{a}^{2} \ddot{z}_{\tau}+\frac{z_{e} A_{2}}{16 g^{2}} \ddot{z}^{3} \\
& +\frac{A_{3} k_{d}}{8 \sqrt{g^{3}}} \dot{z}_{\tau} \ddot{z}^{2}+\frac{A_{3} k_{p}}{8 \sqrt{g^{3}}} \ddot{z}^{2} z_{\tau}+O\left(z_{a}^{4}, \dot{z}^{4}, \ddot{z}^{4}, z_{\tau}^{4}\right) .
\end{aligned}
$$

The linearized system equation is

$$
\dddot{z}+\frac{1}{2} A_{2} z_{e} \ddot{z}+\sqrt{g} A_{3} k_{a} \ddot{z}_{\tau}+\sqrt{g} A_{3} k_{d} \dot{z}_{\tau}+\sqrt{g} A_{3} k_{p} z_{\tau}-A_{2} g z=0 .
$$

The characteristic equation of the linear system (7) is

$$
D(\lambda, \tau)=\lambda^{3}+a \lambda^{2}+b \lambda^{2} e^{-\lambda \tau}+c \lambda e^{-\lambda \tau}+d e^{-\lambda \tau}+e=0,
$$

where

$$
a=\frac{1}{2} A_{2} z_{e}, b=\sqrt{g} A_{3} k_{a}, c=\sqrt{g} A_{3} k_{d}, d=\sqrt{g} A_{3} k_{p}, e=-A_{2} g .
$$

For $\tau=0$ the characteristic equation becomes

$$
D(\lambda, 0)=\lambda^{3}+(a+b) \lambda^{2}+c \lambda+d+e=0 .
$$

Using the Routh-Hurwitz criterion, we obtain the following lemma. 
Lemma 1 ([13]). The eigenvalues of (9) have negative real part if and only if:

$$
a+b>0, d+e>0,(a+b) c>d+e .
$$

From Lemma 1, the maglev system with no delay will be stable if and only if the control parameters satisfy the following conditions

$k_{a}>-\frac{A_{2} z_{e}}{2 A_{3} \sqrt{g}} \stackrel{\text { def }}{=} k_{a_{\text {min }}}, \quad k_{p}>\sqrt{g} \frac{A_{2}}{A_{3}} \stackrel{\text { def }}{=} k_{p_{\min }}, \quad k_{d}>\frac{\sqrt{g} A_{3} k_{p}-A_{2} g}{\left(1 / 2 \cdot A_{2} z_{e}+\sqrt{g} A_{3} k_{a}\right) \sqrt{g} A_{3}} \stackrel{\text { def }}{=} k_{d_{\text {min }}}$.

Let $\lambda=\sigma+i \beta(\beta>0 ; \sigma, \beta \in \mathbb{R})$ be a root of the characteristic equation (8), where $\sigma$ is the growth or decay rate and $\beta$ is the frequency of oscillations. If $\sigma<0$ for every root of (8), then the equilibrium of the system is asymptotically stable and if $\sigma>0$ for at least one root of (8), then the equilibrium of the system is unstable. Thus the stability of the equilibrium may change if there is a root of (8) with $\sigma=0$. Substituting $\lambda=i \beta$ into (8) and separating the real and imaginary parts of (8), we obtain

$$
\begin{aligned}
a \beta^{2}-e & =c \beta \sin \beta \tau-\left(b \beta^{2}-d\right) \cos \beta \tau, \\
\beta^{3} & =c \beta \cos \beta \tau+\left(b \beta^{2}-d\right) \sin \beta \tau .
\end{aligned}
$$

We wish to describe the parameter values for which these equations are satisfied. Eliminating $\sin \beta \tau$ and $\cos \beta \tau$ between (10) and (11), we get an expression for $c$

$$
c=\frac{\sqrt{\left(a \beta^{2}-e\right)^{2}+\beta^{6}-\left(b \beta^{2}-d\right)^{2}}}{\beta} \stackrel{\text { def }}{=} c_{0} .
$$

Recall that $c=\sqrt{g} A_{3} k_{d}$ and $\sqrt{g} A_{3}>0$, so $c$ is directly proportional to the velocity feedback gain, $k_{d}$. For simplicity in our calculations, we use $c$ as the bifurcation parameter. However, in the numerical simulations we give the results in terms of the control parameter $k_{d}$.

From (11), we have $\cos \beta \tau=\left(\beta^{3}-\left(b \beta^{2}-d\right) \sin \beta \tau\right) / c \beta$. Substituting this into (10) and solving for $\tau$, gives

$$
\tau=\frac{1}{\beta}\left[\arcsin \frac{\left(a \beta^{2}-e\right) c \beta+\left(b \beta^{2}-d\right) \beta^{3}}{c^{2} \beta^{2}+\left(b \beta^{2}-d\right)^{2}}+2 j \pi\right], j=0,1,2 \cdots .
$$


For fixed values of the physical parameters $N, r, M, \mu_{0}, z_{e}, S_{0}$ and of the control parameters $k_{p}$ and $k_{a}$, equations (12) and (13) describe curves in the $k_{d}, \tau$ parameter space which are parametrized by $\beta$. Each point of these curves corresponds to a set of parameter values for which the characteristic equation (8) has a pair of pure imaginary roots, $\lambda= \pm i \beta$. The set of values in the parameter space where all the roots of the characteristic equation (8) have negative real part is called the stability region, since the equilibrium point is asymptotically stable inside this region and unstable outside. Parts of the curves defined by equations (12) and (13) form the boundary of this region. The exact boundary will depend on the parameter values considered.

Let us illustrate this with an example. We use realistic values $[11,12]$ for the physical parameters as given in Table 1 . We choose the control parameters $k_{p}$ and $k_{a}$ according to

Table 1: Physical Parameter Values

$N=320, r=0.5 \Omega, M=500 \mathrm{~kg}, \mu_{0}=4 \pi \times 10^{-7}, z_{e}=0.008 \mathrm{~m}, S_{0}=0.047 \mathrm{~m}^{2}$

Lemma 1. With these parameters fixed, we then use (12) and (13) with $j=0$ to plot $k_{d}$ and $\tau$ as $\beta$ is varied. Fig. 2 shows the resulting curves for various values of $k_{p}$ and $k_{a}$. Note that, according to Lemma 1 , for asymptotic stability when $\tau=0, k_{d}$ should be larger than $k_{d_{\min }}$, which depends on $k_{p}$ and $k_{a}$. This value corresponds to the intersection point of each curve with the $k_{d}$ axis. Consideration of equations (12)-(13) shows that each curve approaches the $k_{d}$ axis as $k_{d} \rightarrow \infty$. We do not show the curves with $j>0$ as that they all lie above the corresponding curve with $j=0$ and hence do not form part of the stability boundary. Thus the stability region is the region between each $j=0$ curve and the $k_{d}$ axis. Fig. 2(a) shows the the stability region for $k_{a}$ fixed and varying $k_{p}$. The thin solid curve corresponds to taking $k_{p}=1200$. Increasing $k_{p}$ (dashed line and thick solid line) decreases the size of the stability region. Fig. 2(b) shows the stability region for $k_{p}$ fixed and varying $k_{a}$. The dashed curve corresponds to taking $k_{a}=6$. Increasing $k_{a}$ (thick solid line and thin solid line) increases the size of the stability region. Note that in all cases, the range of values of $k_{d}$ for which the system is stable decreases as $\tau$ increases and there is a critical value of $\tau$, $\tau_{c}$, such that the equilibrium position is unstable for any $k_{d}$ if $\tau>\tau_{c}$. Specifically, $\tau_{c}$ is the 
$\tau$ value at the intersection point, $m$, if it exists, otherwise $\tau_{c}$ is the $\tau$ value at the maximum, $n$. We have tried different pairs of $k_{p}$ and $k_{a}$ and the shapes of the curves defined by (12) and (13) and their behaviour as $k_{p}$ and $k_{a}$ are varied are similar to what is shown in Fig. 2.

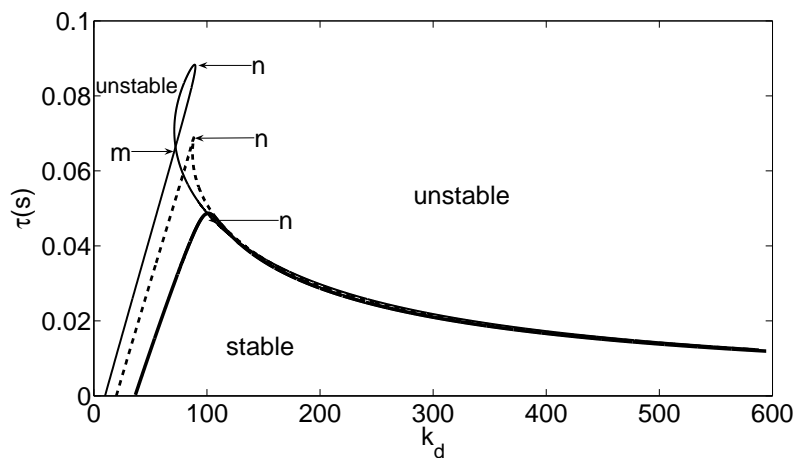

(a) $k_{a}=8$

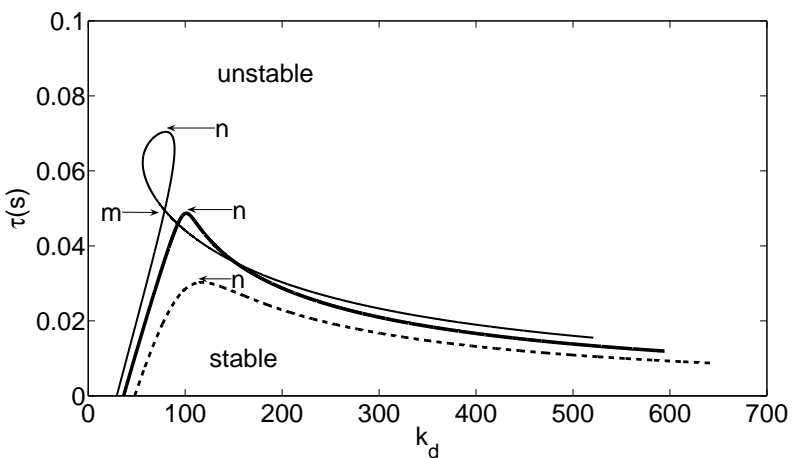

(b) $k_{p}=2000$

Figure 2: Stability region and Hopf bifurcation curve in $k_{d}, \tau$ parameter space. (a) $k_{a}=8$ is fixed, three values of the position feedback parameter: $k_{p}=1200$ (thin, solid line), $k_{p}=1500$ (dashed line), $k_{p}=2000$ (thick, solid line); (b) $k_{p}=2000$ is fixed, three values of the acceleration feedback parameter: $k_{a}=10$ (thin, solid line), $k_{a}=8$ (thick, solid line), $k_{a}=6$ (dashed line).

Note that the points on the curves defined by equations (12) and (13) are potential Hopf bifurcation points. To confirm whether they are we need to check the other conditions of the Hopf Bifurcation Theorem [22, Section 11.1]. For concreteness, we consider the case where $\tau$ is fixed and $k_{d}$ (equivalently, $c$ ) is varied as the bifurcation parameter. The case where $k_{d}$ is fixed and $\tau$ is varied can be analyzed in a similar manner.

First we consider the transversality condition of the Hopf bifurcation theorem, i.e., whether a pair of complex eigenvalues crosses the imaginary axis with non-zero speed. Differentiating (8) with respect to $c$, and evaluating the real part at the bifurcation point gives:

$$
\operatorname{Re}\left[\frac{\mathrm{d} \lambda}{\mathrm{d} c}\right]_{c=c_{0}}^{\lambda=i \beta}=\frac{-\beta q}{p^{2}+q^{2}},
$$

where

$p=-3 \beta^{2} \cos \beta \tau-2 a \beta \sin \beta \tau+b \tau \beta^{2}+c_{0}-d \tau, \quad q=-3 \beta^{2} \sin \beta \tau+2 a \beta \cos \beta \tau-c_{0} \tau \beta+2 b \beta$.

Thus if $q \neq 0$ then a pair of complex conjugate eigenvalues will cross the imaginary axis transversely. Rearranging (10) and (11) and differentiating implicitly with respect to $\beta$ 
shows that

$$
\left[\frac{d \tau}{d \beta}\right]_{c=c_{0}}=\frac{q}{c_{0} \beta^{2}}
$$

Thus we can conclude that the transversality condition is satisfied so long as $\frac{d \tau}{d \beta} \neq 0$. Referring to Fig. 2(b), this means that the transversality condition is satisfied everywhere but at the maximum point $n$.

At points of intersection of the curves defined by equations (12) and (13) there will be two pairs of pure imaginary roots of the characterstic equation (8), and everywhere else on the curves there will only be one root. Thus, away from the intersection points, the nonresonance condition of the Hopf bifurcation is guaranteed to be satisfied. In Fig. 2(b), this means that the nonresonance condition is satisfied everywhere except at the point $m$. Note that this point is potentially a double Hopf bifurcation point.

We thus conclude that at each point $\left(c_{0}, \tau\right)$ on the curves defined by (12) and (13) which

satisfies $\left[\frac{d \tau}{d \beta}\right]_{c=c_{0}} \neq 0$ and which is not a point of intersection of the curves, the trivial solution undergoes a Hopf bifurcation at as $c$ passes through $c_{0}$.

\section{Nonlinear analysis with the method of multiple scales}

In this section, we use the method of multiple scales to seek an approximate periodic solution of equation (6), and hence determine the normal form of the Hopf bifurcation and its type (subcritical or supercritical).

To begin, we introduce a small parameter, $\epsilon$, and a detuning parameter, $\delta$, to describe the closeness of the parameter $c$ to the Hopf bifurcation value $c_{0}$ :

$$
c=c_{0}+\epsilon^{2} \delta
$$

Then, we introduce the fast and slow time scales $T_{0}=t, T_{2}=\epsilon^{2} t$ and expand the solution in terms of the small parameter and the two time scales:

$$
z(t ; \epsilon)=\epsilon z_{1}\left(T_{0}, T_{2}\right)+\epsilon^{2} z_{2}\left(T_{0}, T_{2}\right)+\epsilon^{3} z_{3}\left(T_{0}, T_{2}\right)+\cdots
$$

The solution does not depend on the slow scale $T_{1}=\epsilon t$, because secular terms first appear at $O\left(\epsilon^{3}\right)$. 
In terms of the two time scales, the derivative with respect to $t$ is transformed into

$$
\frac{\mathrm{d}}{\mathrm{d} t}=\frac{\partial}{\mathrm{d} T_{0}}+\epsilon^{2} \frac{\partial}{\partial T_{2}}+\cdots=D_{0}+\epsilon^{2} D_{2}+\cdots .
$$

Using this, we may expand $z(t-\tau)$ in terms of $\epsilon$ and the two time scales:

$$
\begin{aligned}
z(t-\tau ; \epsilon) & =\sum_{m=1}^{3} \epsilon^{m} z_{m}\left(T_{0}-\tau, T_{2}-\epsilon^{2} \tau\right)+\cdots \\
& =\sum_{m=1}^{3} \epsilon^{m} z_{m}\left(T_{0}-\tau, T_{2}\right)-\epsilon^{3} \tau D_{2} z_{1}\left(T_{0}-\tau, T_{2}\right)+\cdots .
\end{aligned}
$$

Substituting eqs. (15)-(17) into eq. (6), expanding the result in $\epsilon$, and equating coefficients of like powers of $\epsilon$, we obtain

$$
\begin{aligned}
O\left(\epsilon^{1}\right): & D_{0}^{3} z_{1}+a D_{0}^{2} z_{1}+b D_{0}^{2} z_{1 \tau}+c_{0} D_{0} z_{1 \tau}+d z_{1 \tau}+e z_{1}=0 \\
O\left(\epsilon^{2}\right): \quad & D_{0}^{3} z_{2}+a D_{0}^{2} z_{2}+b D_{0}^{2} z_{2 \tau}+c_{0} D_{0} z_{2 \tau}+d z_{2 \tau}+e z_{2}=\frac{A_{3} k_{a}}{2 \sqrt{g}} D_{0}^{2} z_{1} \cdot D_{0}^{2} z_{1 \tau}+ \\
& \frac{A_{2} z_{e}}{8 g}\left(D_{0}^{2} z_{1}\right)^{2}+\frac{c_{0}}{2 g} D_{0} z_{1 \tau} \cdot D_{0}^{2} z_{1}+\frac{A_{3} k_{p}}{2 \sqrt{g}} D_{0}^{2} z_{1} \cdot z_{1 \tau}-A_{2} z_{1} \cdot D_{0}^{2} z_{1} \\
O\left(\epsilon^{3}\right): \quad & D_{0}^{3} z_{3}+3 D_{0}^{2} D_{2} z_{1}+a D_{0}^{2} z_{3}+2 a D_{0} D_{2} z_{1}+b D_{0}^{2} z_{3 \tau}-b \tau D_{0}^{2} D_{2} z_{1 \tau}+2 b D_{0} D_{2} z_{1 \tau} \\
& +c_{0} D_{0} z_{3 \tau}-c_{0} \tau D_{0} D_{2} z_{1 \tau}+c_{0} D_{2} z_{1 \tau}+\delta D_{0} z_{1 \tau}+d z_{3 \tau}-d \tau D_{2} z_{1 \tau}+e z_{3}= \\
& \frac{A_{3} k_{a}}{2 \sqrt{g}}\left(D_{0}^{2} z_{1} \cdot D_{0}^{2} z_{2 \tau}+D_{0}^{2} z_{2} \cdot D_{0}^{2} z_{1 \tau}\right)+\frac{A_{2} z_{e}}{4 g} D_{0}^{2} z_{1} \cdot D_{0}^{2} z_{2}+\frac{c_{0}}{2 g}\left(D_{0} z_{1 \tau} \cdot D_{0}^{2} z_{2}+\right. \\
& \left.D_{0} z_{2 \tau} \cdot D_{0}^{2} z_{1}\right)+\frac{A_{3} k_{p}}{2 \sqrt{g}}\left(D_{0}^{2} z_{1} \cdot z_{2 \tau}+D_{0}^{2} z_{2} \cdot z_{1 \tau}\right)-A_{2}\left(z_{1} \cdot D_{0}^{2} z_{2}+z_{2} \cdot D_{0}^{2} z_{1}\right)+ \\
& \frac{A_{3} k_{a}}{8 \sqrt{g^{3}}}\left(D_{0}^{2} z_{1}\right)^{2} \cdot D_{0}^{2} z_{1 \tau}+\frac{A_{2} z_{e}}{16 g^{2}}\left(D_{0}^{2} z_{1}\right)^{3}+\frac{c_{0}}{8 g^{2}} D_{0} z_{1 \tau} \cdot\left(D_{0}^{2} z_{1}\right)^{2}+\frac{A_{3} k_{p}}{8 \sqrt{g^{3}}}\left(D_{0}^{2} z_{1}\right)^{2} z_{1}(21)
\end{aligned}
$$

The solution of (19) corresponding to the roots $\pm i \beta$ of (8) can be expressed in the form

$$
z_{1}\left(T_{0}, T_{2}\right)=A\left(T_{2}\right) e^{i \beta T_{0}}+\bar{A}\left(T_{2}\right) e^{-i \beta T_{0}}
$$

where $A\left(T_{2}\right)$, which is determined by eliminating the secular terms at $O\left(\epsilon^{3}\right)$, is a complexvalued function of $T_{2}$, and $\bar{A}\left(T_{2}\right)$ is its complex conjugate. Note that $\beta$ is the critical frequency corresponding to $c_{0}$.

Substituting (22) into (20) yields

$$
D_{0}^{3} z_{2}+a D_{0}^{2} z_{2}+b D_{0}^{2} z_{2 \tau}+c_{0} D_{0} z_{2 \tau}+d z_{2 \tau}+e z_{2}=R A^{2}\left(T_{2}\right) e^{2 i \beta T_{0}}+R A\left(T_{2}\right) \bar{A}\left(T_{2}\right)+\mathrm{cc},
$$


where cc represents the complex conjugate of the preceding terms and

$$
\begin{aligned}
R & =R_{r}+i R_{i} \\
& =\left(\frac{A_{3} k_{a}}{2 \sqrt{g}} \beta^{4} \cos \beta \tau+\frac{A_{2} z_{e}}{8 g} \beta^{4}-\frac{c_{0}}{2 g} \beta^{3} \sin \beta \tau-\frac{A_{3} k_{p}}{2 \sqrt{g}} \beta^{2} \cos \beta \tau+A_{2} \beta^{2}\right) \\
& +i\left(-\frac{A_{3} k_{a}}{2 \sqrt{g}} \beta^{4} \sin \beta \tau-\frac{c_{0}}{2 g} \beta^{3} \cos \beta \tau+\frac{A_{3} k_{p}}{2 \sqrt{g}} \beta^{2} \sin \beta \tau\right) .
\end{aligned}
$$

The particular solution of eq. (23) can be expressed as

$$
z_{2}=P A^{2} e^{2 i \beta T_{0}}+Q A \bar{A}+\mathrm{cc}
$$

where

$$
\begin{array}{rlrl}
P & =P_{r}+i P_{i} & Q & =Q_{r}+i Q_{i} \\
& =\frac{R_{r} S_{r}+R_{i} S_{i}}{S_{r}^{2}+S_{i}^{2}}+i \frac{-R_{r} S_{i}+R_{i} S_{r}}{S_{r}^{2}+S_{i}^{2}} & & =\frac{R_{r}}{d+e}+i \frac{R_{i}}{d+e}
\end{array}
$$

with

$$
\begin{aligned}
S & =S_{r}+i S_{i} \\
& =\left(-4 a \beta^{2}-4 b \beta^{2} \cos 2 \beta \tau+2 c_{0} \beta \sin 2 \beta \tau+e+d \cos 2 \beta \tau\right) \\
& +i\left(-8 \beta^{3}+4 b \beta^{2} \sin 2 \beta \tau+2 c_{0} \beta \cos 2 \beta \tau-d \sin 2 \beta \tau\right) .
\end{aligned}
$$

Substituting (22) and (24) into equation (21) and eliminating the secular terms, we get the complex-valued normal form of the Hopf bifurcation

$$
M A^{\prime}=-i \delta \beta A e^{-i \beta \tau}+\Gamma A^{2} \bar{A}
$$


where $M$ and $\Gamma$ are given as follows

$$
\begin{aligned}
M= & M_{r}+i M_{i} \\
= & \left(-3 \beta^{2}+b \beta^{2} \tau \cos \beta \tau+2 b \beta \sin \beta \tau-c_{0} \tau \beta \sin \beta \tau+c_{0} \cos \beta \tau-d \tau \cos \beta \tau\right) \\
& +i\left(2 a \beta-b \beta^{2} \tau \sin \beta \tau+2 b \beta \cos \beta \tau-c_{0} \tau \beta \cos \beta \tau-c_{0} \sin \beta \tau+d \tau \sin \beta \tau\right) \\
\Gamma= & \Gamma_{r}+i \Gamma_{i} \\
= & {\left[\frac{A_{3} k_{a}}{2 \sqrt{g}}\left(4 \beta^{4} P_{r} \cos 2 \beta \tau+4 \beta^{4} P_{i} \sin 2 \beta \tau+4 \beta^{4} P_{r} \cos \beta \tau-4 \beta^{4} P_{i} \sin \beta \tau\right)+\frac{A_{2} z_{e}}{g} \beta^{4} P_{r}+\right.} \\
& \frac{c_{0}}{2 g}\left(-4 \beta^{3} P_{r} \sin \beta \tau-4 \beta^{3} P_{i} \cos \beta \tau-2 \beta^{3} P_{r} \sin 2 \beta \tau+2 \beta^{3} P_{i} \cos 2 \beta \tau\right)+\frac{A_{3} k_{p}}{2 \sqrt{g}}\left(-2 \beta^{2} Q_{r}\right. \\
& \left.-\beta^{2} P_{r} \cos 2 \beta \tau-\beta^{2} P_{i} \sin 2 \beta \tau-4 \beta^{2} P_{r} \cos \beta \tau+4 \beta^{2} P_{i} \sin \beta \tau\right)+A_{2}\left(5 \beta^{2} P_{r}+2 \beta^{2} Q_{r}\right) \\
& \left.-\frac{A_{3} k_{a}}{8 \sqrt{g^{3}}} 3 \beta^{6} \cos \beta \tau-\frac{A_{2} z_{e}}{16 g^{2}} 3 \beta^{6}+\frac{c_{0}}{8 g^{2}} 3 \beta^{5} \sin \beta \tau+\frac{A_{3} k_{p}}{8 \sqrt{g^{3}}} 3 \beta^{4} \cos \beta \tau\right] \\
& +i\left[\frac{A_{3} k_{a}}{2 \sqrt{g}}\left(4 \beta^{4} P_{i} \cos 2 \beta \tau-4 \beta^{4} P_{r} \sin 2 \beta \tau+4 \beta^{4} P_{i} \cos \beta \tau+4 \beta^{4} P_{r} \sin \beta \tau\right)+\frac{A_{2} z_{e}}{g} \beta^{4} P_{i}\right. \\
& \frac{c_{0}}{2 g}\left(4 \beta^{3} P_{r} \cos \beta \tau-4 \beta^{3} P_{i} \sin \beta \tau-2 \beta^{3} P_{r} \cos 2 \beta \tau-2 \beta^{3} P_{i} \sin 2 \beta \tau\right) \\
& +\frac{A_{3} k_{p}}{2 \sqrt{g}}\left(-\beta^{2} P_{i} \cos 2 \beta \tau+\beta^{2} P_{r} \sin 2 \beta \tau-4 \beta^{2} P_{i} \cos \beta \tau-4 \beta^{2} P_{r} \sin \beta \tau\right)+A_{2}-5 \beta^{2} P_{i} \\
& \left.+\frac{A_{3} k_{a}}{8 \sqrt{g^{3}}} \beta^{6} \sin \beta \tau+\frac{c_{0}}{8 g^{2}} \beta^{5} \cos \beta \tau-\frac{A_{3} k_{p}}{8 \sqrt{g^{3}}} \beta^{4} \sin \beta \tau\right] .
\end{aligned}
$$

Next, we express $A\left(T_{2}\right)$ in the polar form

$$
A\left(T_{2}\right)=\frac{1}{2} \alpha\left(T_{2}\right) e^{i \omega\left(T_{2}\right)}
$$

where $\alpha\left(T_{2}\right)$ and $\omega\left(T_{2}\right)$ are real-valued functions of the slow time $T_{2}$. Substituting (26) into (25) yields

$$
\begin{aligned}
\alpha^{\prime} & =\delta \chi_{1} \alpha+\chi_{3} \alpha^{3}, \\
\omega^{\prime} & =\delta \chi_{2}+\chi_{4} \alpha^{2},
\end{aligned}
$$

where

$$
\begin{array}{ll}
\chi_{1}=\frac{-\beta}{M_{r}^{2}+M_{i}^{2}}\left(\sin \beta \tau M_{r}+\cos \beta \tau M_{i}\right), & \chi_{3}=\frac{\Gamma_{r} M_{r}+\Gamma_{i} M_{i}}{4\left(M_{r}^{2}+M_{i}^{2}\right)}, \\
\chi_{2}=\frac{\beta}{M_{r}^{2}+M_{i}^{2}}\left(\sin \beta \tau M_{i}-\cos \beta \tau M_{r}\right), & \chi_{4}=\frac{\Gamma_{i} M_{r}-\Gamma_{r} M_{i}}{4\left(M_{r}^{2}+M_{i}^{2}\right)} .
\end{array}
$$

A periodic solution of eq. (6) must yield $\alpha \neq 0$ and $D_{2} \alpha=0$, namely

$$
\alpha=\sqrt{\frac{-\delta \chi_{1}}{\chi_{3}}} .
$$


Substituting (31) into (28) yields

$$
\omega=\epsilon^{2}\left(\chi_{2}-\delta \chi_{1} \chi_{4} / \chi_{3}\right) t+\omega_{0}
$$

where $\omega_{0}$ is a constant. Returning to the original variables, we obtain following expression for the limit cycle

$$
z(t)=\epsilon \sqrt{\frac{-\delta \chi_{1}}{\chi_{3}}} \cos \left[\beta t+\epsilon^{2}\left(\chi_{2}-\frac{\delta \chi_{1} \chi_{4}}{\chi_{3}}\right) t+\omega_{0}\right]+O\left(\epsilon^{2}\right) .
$$

The stability of this solution is determined by the derivative of the right-hand side of equation (27) evaluated on the limit cycle, i.e.,

$$
\left[\delta \chi_{1}+3 \chi_{3} \alpha^{2}\right]_{\alpha=\sqrt{-\delta \chi_{1} / \chi_{3}}}=-2 \delta \chi_{1}
$$

Thus limit cycle will be stable (unstable) if $\delta \chi_{1}>0(<0)$. Since the limit cycle exists only when $-\delta \chi_{1} / \chi_{3}>0$, the stability of the limit cycle is determine by the sign of $\chi_{3}$, i.e. the limit cycle will be stable (unstable) if $\chi_{3}<0(>0)$.

Comparing eqs. (14) and (29) shows that $\chi_{1}=\left.\operatorname{Re}\left[\frac{d \lambda}{d c}\right]\right|_{c=c_{0}} ^{\lambda=i \beta}$, as expected from the theory. Thus the equilibrium point will lose (gain) stability as $c$ increases through $c_{0}$ if $\chi_{1}>0(<0)$.

In summary, we have the following. If the signs of $\chi_{1}$ and $\chi_{3}$ are the same, then the limit cycle exists when $\delta<0$, i.e., $c<c_{0}$. The limit cycle will be stable (unstable) and the Hopf bifurcation supercritical (subcritical) when $\chi_{1}<0, \chi_{3}<0\left(\chi_{1}>0, \chi_{3}>0\right)$. If the signs of $\chi_{1}$ and $\chi_{3}$ are different, then the limit cycle exists when $\delta>0$, i.e., $c>c_{0}$. The limit cycle will stable (unstable) and the Hopf bifurcation supercritical (subcritical) if $\chi_{1}>0, \chi_{3}<0$ $\left(\chi_{1}<0, \chi_{3}>0\right)$.

We now apply the results above to determine the criticality of the Hopf bifurcation curves for some specific values of the parameters. Recall from Fig. 2, that for each fixed value of $\tau$, the system has two different Hopf bifurcation values of the velocity gain: $k_{d 1}<k_{d 2}$. The equilibrium state of the maglev system is asymptotically stable if $k_{d} \in\left(k_{d 1}, k_{d 2}\right)$, otherwise it is unstable. From the stability of the equilibrium state, it is clear that $\chi_{1}<0$ at $k_{d 1}$ and $\chi_{1}>0$ at $k_{d 2}$. Further, as discussed above, the criticality of the Hopf bifurcation and stability of the associated limit cycle are determined by the sign of $\chi_{3}$. We evaluated $\chi_{3}$ at 


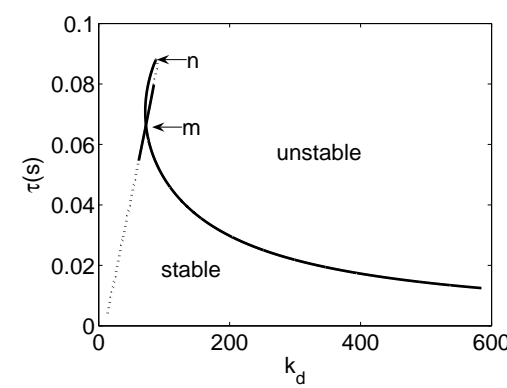

(a) $k_{p}=1200, k_{a}=8$

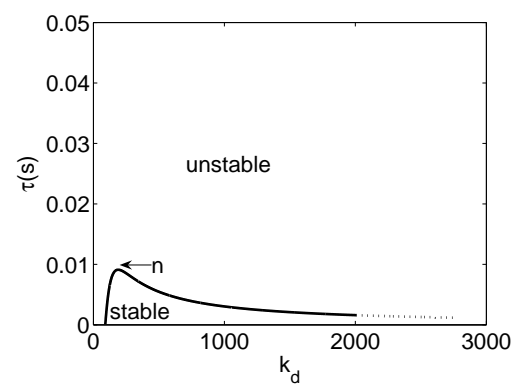

(b) $k_{p}=2000, k_{a}=3$

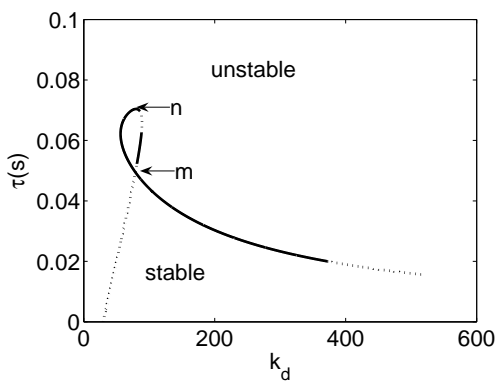

(c) $k_{p}=2000, k_{a}=10$

Figure 3: Hopf bifurcation curves in the $k_{d}, \tau$ parameter space. A solid line indicates $\chi_{3}<0$, and a dashed line indicates $\chi_{3}>0$. Physical parameters are as in Table 1. Feedback parameters are as indicated. Note that (b) has a different scale than (a) and (c). The stability region of the equilibrium point is indicated.

each point of the Hopf bifurcation curves for three pairs of $k_{p}$ and $k_{a}$. The results are shown in Fig. 3.

Since there are two Hopf bifurcations which can be either subcritical or supercritical, there are four possible branching types as shown in Fig. 4. The system exhibits all of these as we now describe. For Fig. 3(a) $\left(k_{p}=1200, k_{a}=8\right)$, the branching type is as in Fig. 4(a)

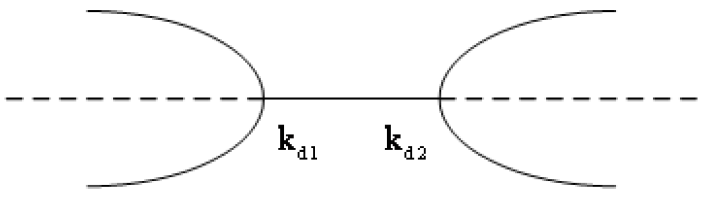

(a)

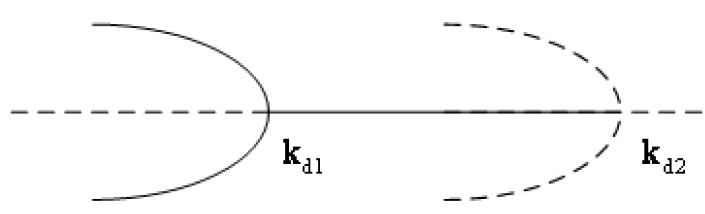

(c)

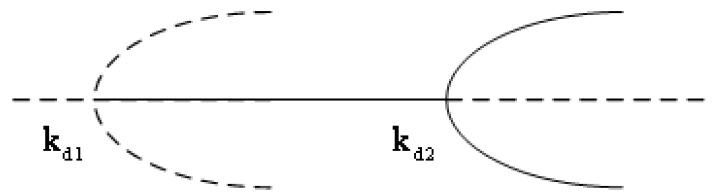

(b)

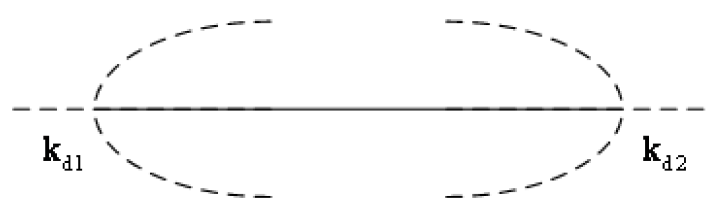

(d)

Figure 4: Different branching structure of the Hopf bifurcations according to the signs of $\chi_{1}$ and $\chi_{3}$.

when $0.055<\tau<\tau_{c}$ and as in Fig. 4(b) when $\tau<0.055$. (Recall that $\tau_{c}$ is the the largest value of $\tau$ for which the equilibrium state is stable). For Fig. 3(b) ( $\left.k_{p}=2000, k_{a}=3\right)$, the branching type is as in Fig. 4(a) when $0.0016<\tau<\tau_{c}$ and Fig. 4(c) when $\tau<0.0016$. For Fig. 3(c) $\left(k_{p}=2000, k_{a}=10\right)$, the branch type is as in Fig. 4(b) when $0.02<\tau<\tau_{c}$ and as in Fig. 4(d) when $\tau<0.02$. Note that at points where the criticality changes, the Hopf 
bifurcation is degenerate. It has been shown $[23,24]$ that a secondary bifurcation curve may emerge from such points. This secondary bifurcation is a saddle node bifurcation of limit cycles which produces a second, large amplitude limit cycle in addition to the one produced by the Hopf bifurcation.

\section{Numerical studies}

In this section we consider the maglev model (3)-(4) with the physical parameters given in Table 1 and the control feedback gains $k_{p}=2000$ and $k_{a}=10$. The Hopf bifurcation curve is shown in Fig. 3(c). We will focus on the case when $\tau=0.047$. Then $k_{d 1} \approx 76.61$, $k_{d 2} \approx 87.75$ and the bifurcations are as shown in Fig. 4(b), i.e., the Hopf bifurcation at $k_{d 1}$ is subcritical and that at $k_{d 2}$ is supercritical. We will compare these theoretical results with numerical simulations and numerical continuation studies of the model (3)-(4), with parameter values as indicated above. The initial conditions used in the numerical simulations are of the form $(z(t), \dot{z}(t), \ddot{z}(t))=\left(z_{0}, 0,0\right),-\tau \leq t \leq 0$ for various values of $z_{0}$.

From Fig. 5 (a)-(b), if $k_{d}=75<k_{d 1} \approx 76.61$, solutions diverge from the trivial solution, i.e., it is unstable; if $k_{d}=78>k_{d 1}$ and $z_{0}=0.001 \mathrm{~m}$, solutions approach the trivial solution, indicating it is asymptotically stable (see Fig. 5 (c)-(d)). However, if we choose $k_{d}=78$ and a larger initial condition, $z_{0}=0.022 \mathrm{~m}$, solutions diverge (see Fig. 5 (e)-(f)), which suggests that an unstable limit cycle exists when $k_{d}>k_{d 1}$ and that the Hopf bifurcation is subcritical.

From Fig. 6 (a)-(b), if $k_{d}=85<k_{d 2} \approx 87.75$, the trivial solution is stable; if we choose the same control gain $k_{d}=85$ but a larger initial condition $z_{0}=0.0328 \mathrm{~m}$ (larger than the initial condition in Fig. 5 (e)-(f)), Fig. 6 (c)-(d) show the position variable grows quickly, which suggests the existence of an unstable limit cycle. From Fig. 6 (e)-(f), if $k_{d}=90>k_{d 2}$, the trivial solution is unstable and a stable limit cycle exists, indicating that the Hopf bifurcation is supercritical; if we choose the same $k_{d}=90$ but a larger initial condition $z_{0}=0.033 \mathrm{~m}$ (larger than the initial condition in Fig. 6 (c)-(d)), the solution grows rapidly (see Fig. 6 (g)-(h)), which suggests the existence of an unstable limit cycle when $k_{d}>k_{d 2}$.

The horizontal lines in Fig. 5 and Fig. 6. indicate where $z=-z_{e}$, i.e., the air-gap between the train and the rail becomes zero. The model becomes invalid at this point as it does not include the effect of the impact. In Fig. 5 (a),(e) and Fig. 6 (c),(g) one can see that the 


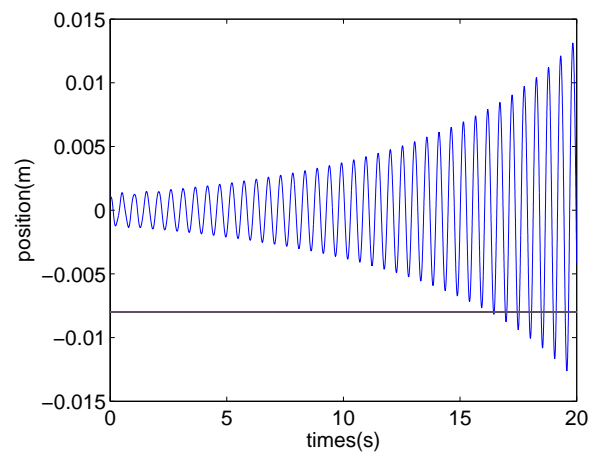

(a) $k_{d}=75, z_{0}=0.001 m$

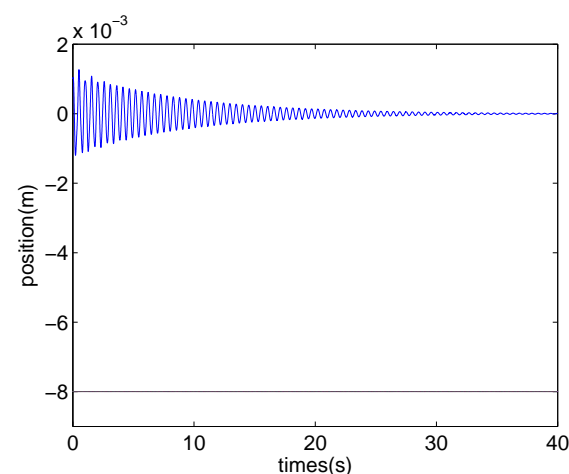

(c) $k_{d}=78, z_{0}=0.001 \mathrm{~m}$

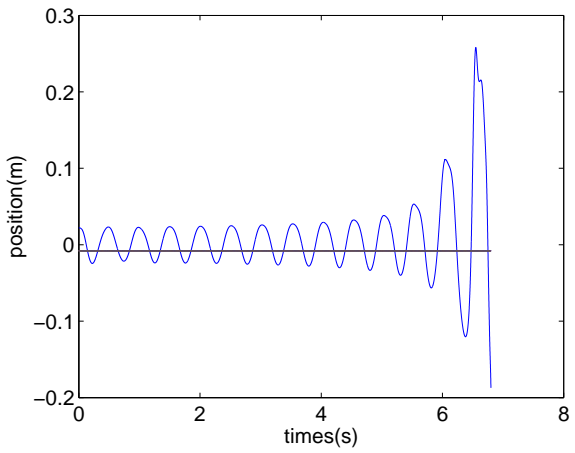

(e) $k_{d}=78, z_{0}=0.022 m$

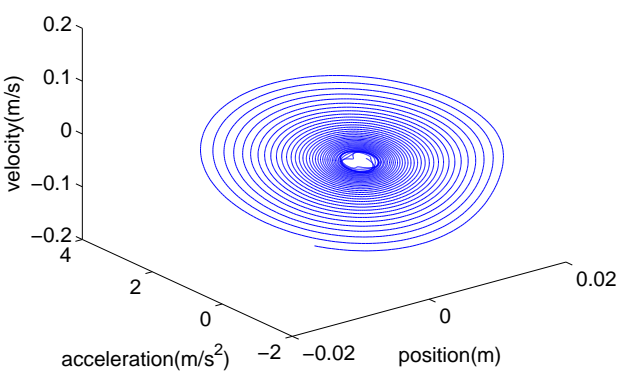

(b) $k_{d}=75, z_{0}=0.001 m$

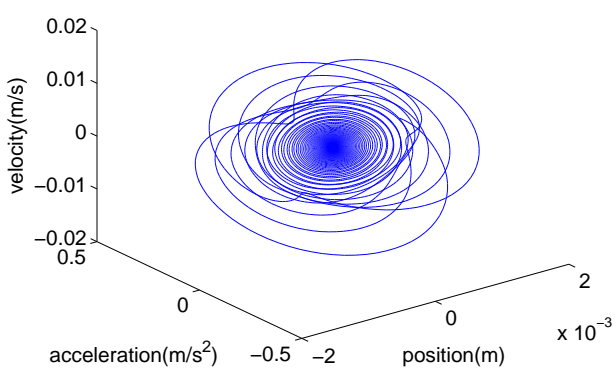

(d) $k_{d}=78, z_{0}=0.001 m$

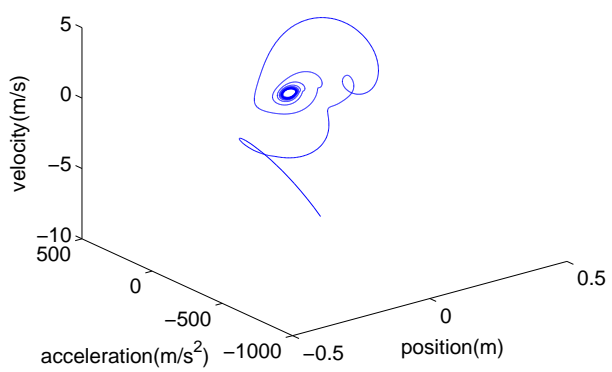

(f) $k_{d}=78, z_{0}=0.022 m$

Figure 5: Numerical simulations of the maglev system (5), using the initial condition $(z(t), \dot{z}(t), \ddot{z}(t))=$ $\left(z_{0}, 0,0\right),-\tau \leq t \leq 0$, with values of $z_{0}$ as indicated. Physical parameters are as in Table 1 . Feedback parameters are $k_{p}=2000, k_{a}=10, \tau=0.047$ and $k_{d}$ as indicated. (a),(c),(e) are trajectories of $z$ vs time and (b),(d),(f) are (pseudo) phase portraits. The horizontal lines in (a),(c),(d) indicate where air-gap becomes zero. 


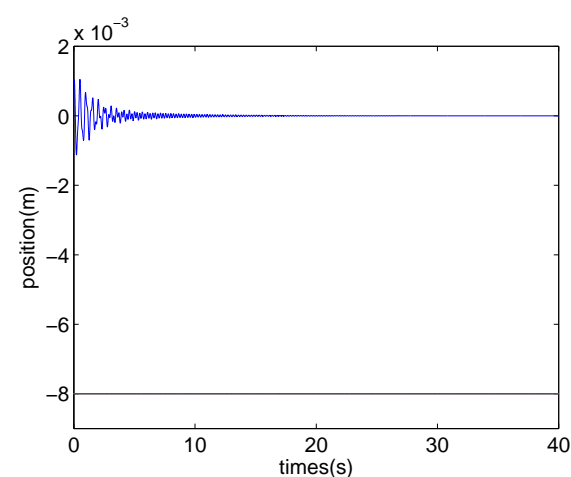

(a) $k_{d}=85, z_{0}=0.001 m$

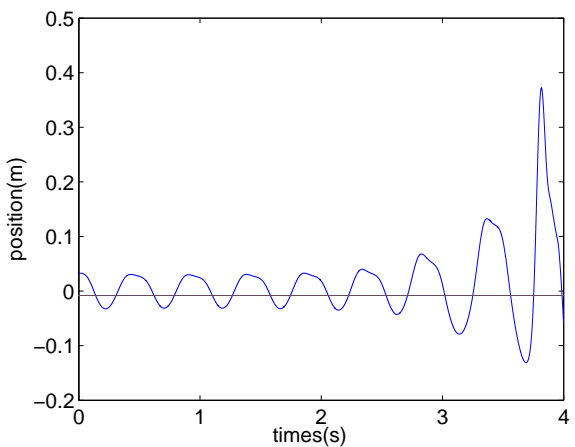

(c) $k_{d}=85, z_{0}=0.0328 m$

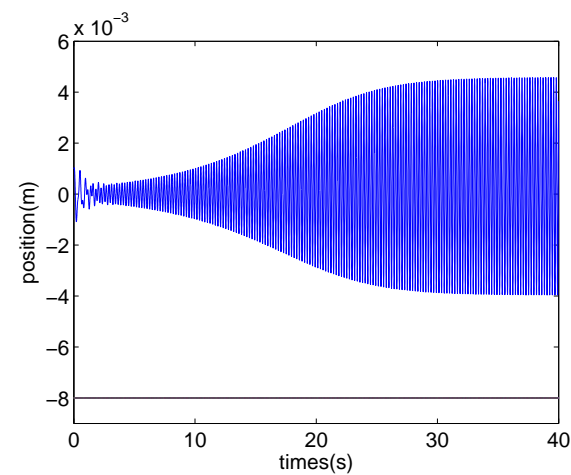

(e) $k_{d}=90, z_{0}=0.001 m$

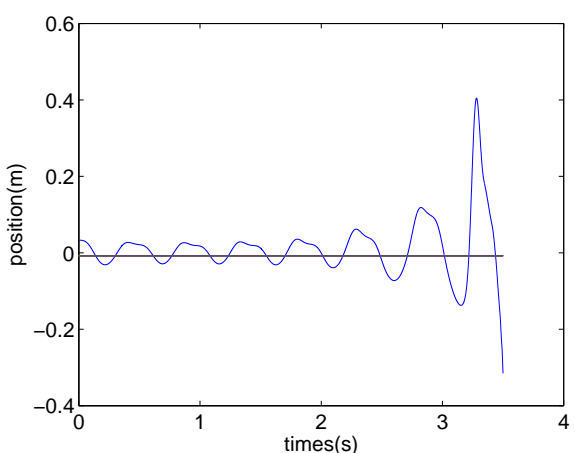

(g) $k_{d}=90, z_{0}=0.033 m$

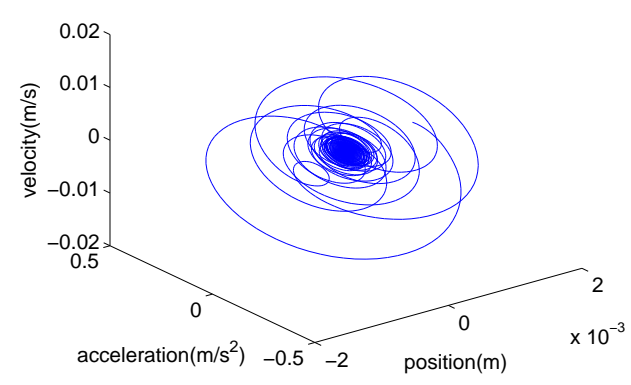

(b) $k_{d}=85, z_{0}=0.001 m$

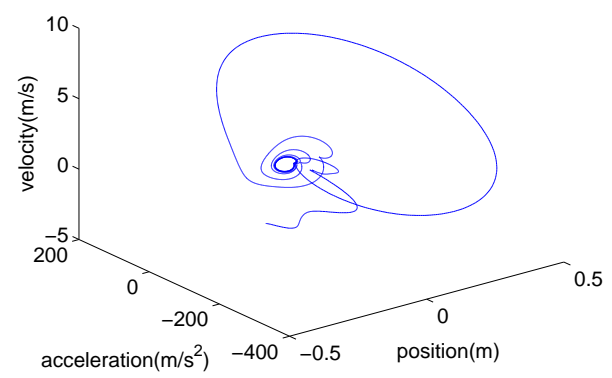

(d) $k_{d}=85, z_{0}=0.0328 m$

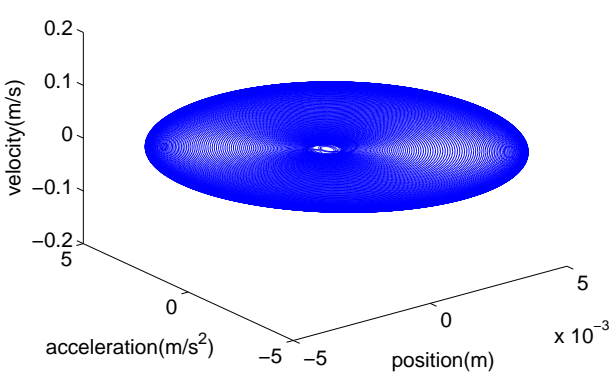

(f) $k_{d}=90, z_{0}=0.001 \mathrm{~m}$

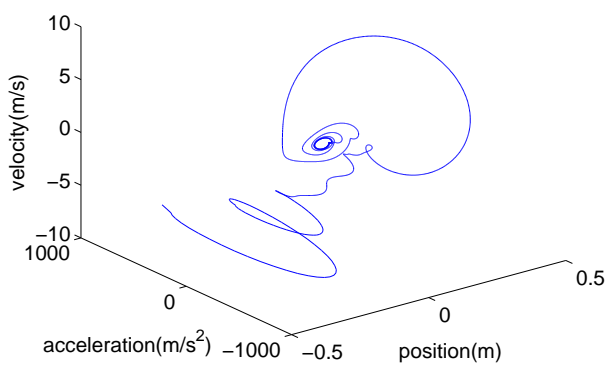

(h) $k_{d}=90, z_{0}=0.033 \mathrm{~m}$

Figure 6: Numerical simulations of the maglev system (5), using the initial condition $(z(t), \dot{z}(t), \ddot{z}(t))=$ $\left(z_{0}, 0,0\right),-\tau \leq t \leq 0$, with values of $z_{0}$ as indicated. Physical parameters are as in Table 1. Feedback parameters are $k_{p}=2000, k_{a}=10, \tau=0.047$ and $1 k^{2}$ as indicated. (a),(c),(e),(g) are trajectories of $z$ vs time; and (b),(d),(f),(h) are (pseudo) phase portraits. The horizontal lines in (a),(c),(d),(g) indicate where air-gap becomes zero. 


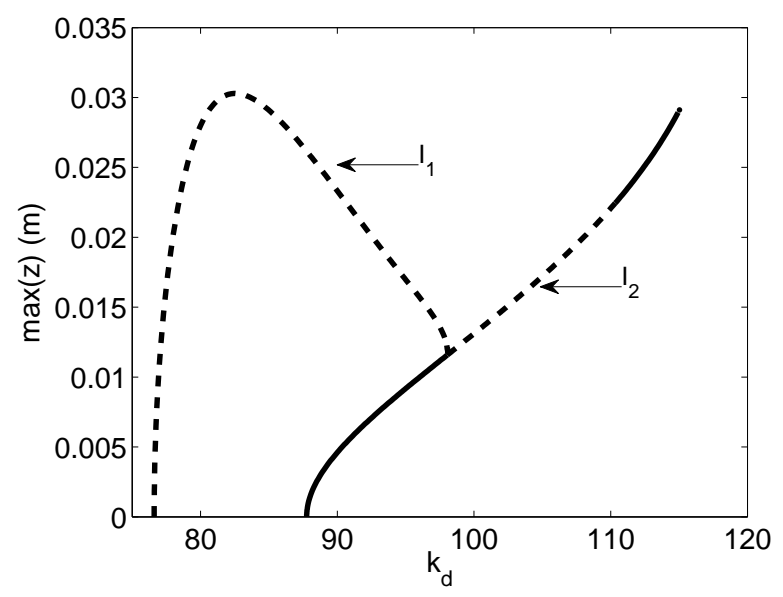

Figure 7: Numerical continuation of the periodic orbits of (5) with $k_{p}=2000, k_{a}=10, \tau=0.047$ and physical parameters as given in Table 1. Dashed/solid lines indicate unstable/stable periodic orbits.

instability causes the train to hit the rail. However, in Fig. 6 the amplitude of the oscillations is small enough that the train does not hit the rail.

Thus the numerical simulations agree with the local bifurcation diagram of Fig. 4(b) as predicted by the theory. They also suggest that the unstable limit cycle created in the subcritical Hopf bifurcation at $k_{d}=k_{d 1}$ persists throughout the entire range of values where the equilibrium point is stable, $\left(k_{d 1}, k_{d 2}\right)$. To verify this, we carried out a numerical continuation study of the periodic orbits using the software DDE-BIFTOOL [25]. The result is shown in Fig. 7. This confirms that the unstable limit cycle created by the Hopf bifurcation at $k_{d}=k_{d 1}(\approx 76.61)$ persists past the Hopf bifurcation at $k_{d}=k_{d 2}(\approx 87.75)$. Note that the limit cycle produced by the Hopf bifurcation at $k_{d 1}\left(l_{1}\right.$ in Fig. 7$)$ terminates on the limit cycle produce by the Hopf bifurcation at $k_{d 2}$ in what appears to be a period doubling bifurcation. Numerical simulations for parameter values near this bifurcation point show transient oscillations which look similar to typical period two orbits. The bifurcation diagram of Fig. 7) may be explained by noting that the frequencies associated with the double Hopf bifurcation at the intersection point $\left(k_{d}, \tau\right) \approx(100,0.05)$ are close to 1:2 resonance. The bifurcation diagram of Fig. 7 has been predicted to occur near such 1:2 resonant double Hopf bifurcation points $[26,27]$.

We have performed numerical simulations and numerical continuation studies for several 
other values of $\tau$. These also agree with the predictions of the nonlinear analysis. In particular, we observe for $\tau<0.02$ that the bifurcation at $k_{d 2}$ becomes subcritical, as predicted by the nonlinear analysis. In all cases, it appears that an unstable limit cycle coexists with the stable equilibrium point for $k_{d 1}<k_{d}<k_{d 2}$.

A valid question is whether the behaviour we observe persists if other control gains are varied. To partially address this question, we performed some studies with the delay fixed at the value above, $\tau=0.047 \mathrm{~s}$. With fixed delay, the stability region can be visualized in a parameter space consisting of two of the feedback gains. The boundary of the region of stability in these parameters is easily found by solving (10)-(11) for $k_{d}$ and $k_{a}$ or $k_{p}$ in terms of the other parameters:

$$
\begin{aligned}
k_{d} & =\frac{1}{\sqrt{g} A_{3} \beta}\left(\left(a \beta^{2}-e\right) \sin (\beta \tau)+\beta^{3} \cos (\beta \tau)\right) \\
k_{p}-k_{a} \beta^{2} & =\frac{1}{\sqrt{g} A_{3}}\left(\left(a \beta^{2}-e\right) \cos (\beta \tau)-\beta^{3} \sin (\beta \tau)\right)
\end{aligned}
$$

The resulting boundaries with parameters as in the example above are shown in Figure 8. In both cases, the stability region is a closed region of the plane. Note that the thin lines define the stability region for $\tau=0$, thus one effect of the delay is to reduce the choices of feedback gains for which the equilibrium point will be asymptotically stable. Similar stability regions have been observed for models of the inverted pendulum with time delayed feedback $[31,32,33,34]$. Numerical continuation studies varying $k_{p}$ or $k_{a}$ with $k_{d}$ fixed are shown in

Figure 9. In both cases there are two branches of periodic orbits emanating from the Hopf bifurcations which occur on the boundary of the stability region. In both cases, the unstable limit cycle created by the subcritical Hopf persists throughout the region of stability of the equilibrium point. The branching structure when $k_{a}$ is varied is very similar to that when $k_{d}$ is varied.

\section{Conclusions and future research}

In this paper, the stability and Hopf bifurcation of the suspension system of a maglev train with time-delayed position, velocity and acceleration feedback are studied.

We first described a method for choosing appropriate control gains to stabilize the system. Given values of the physical parameters in the system, the position and acceleration feedback 

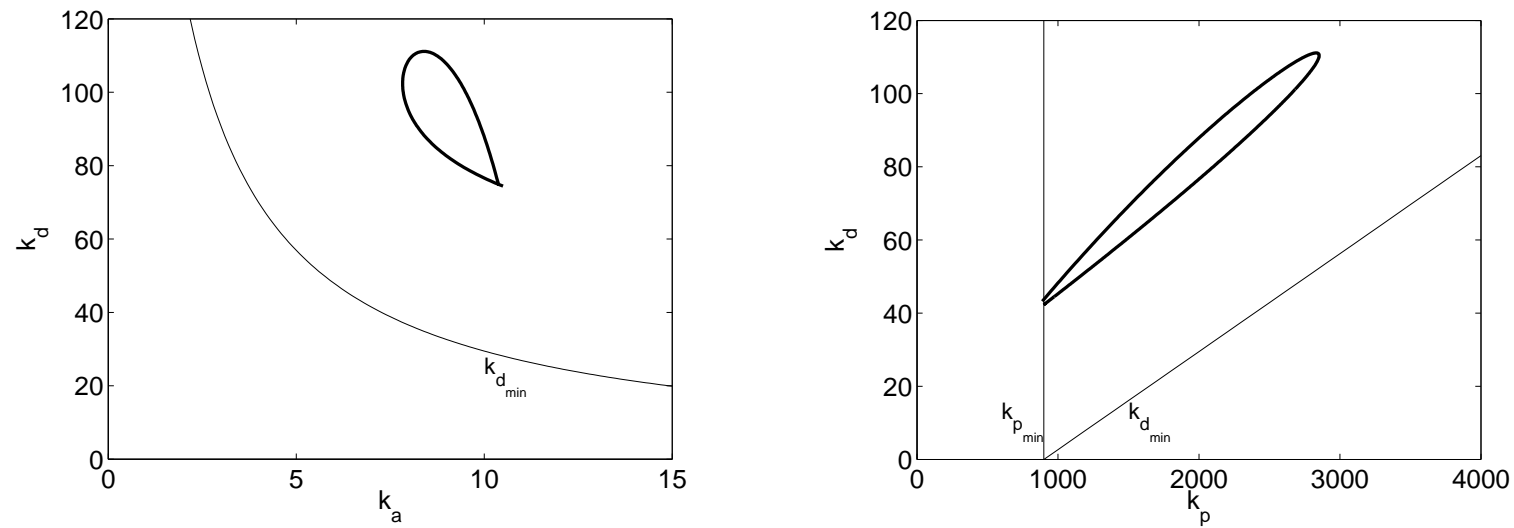

Figure 8: Stability region in (a) $k_{d}, k_{a}$ (b) $k_{d}, k_{p}$ parameter space. Physical parameters are as given in Table 1, $\tau=0.047$ and (a) $k_{p}=2000$ (b) $k_{a}=10$. The thick line is the boundary defined by (35). The thin lines are $k_{a m i n}, k_{p m i n}$ and $k_{d m i n}$ as defined by Lemma 1 .
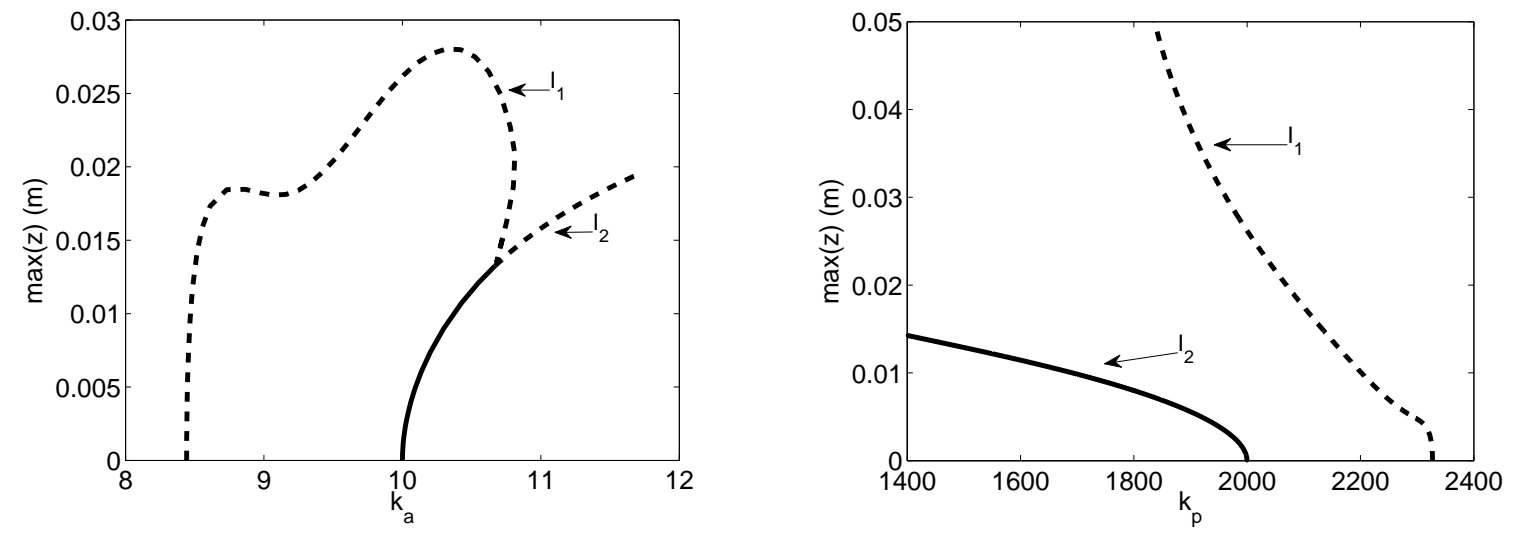

Figure 9: Numerical continuation of the periodic orbits of (5) with $k_{d}=87.75, \tau=0.047$ and physical parameters as given in Table 1. (a) $k_{p}=2000$ (b) $k_{a}=10$. Dashed/solid lines indicate unstable/stable periodic orbits. The equilibrium point is stable between starting points of the two branches. 
gains can be chosen using the Routh-Hurwitz criterion. A set of values of the delay and the velocity feedback gain for which the the maglev train is stable can then be described. For the parameter values we investigated, increasing the acceleration feedback gain or decreasing the position feedback gain increased the size of this set of values. Further, there is always a critical delay such that if the delay is larger than the critical delay there is no value of the velocity feedback gain, $k_{d}$, that stabilizes the system. For any fixed delay less than the critical delay there is a finite range of values $\left(k_{d 1}, k_{d 2}\right)$ such that any feedback gain in this range stabilizes the system. The smaller the delay, the larger this range. A similar effect of the delay on the choice of feedback gains for which the system may be stabilized has been observed in models for an inverted pendulum with time delayed feedback [31, 32, 33, 34].

We next showed that these critical velocity feedback gains, $k_{d 1}$ and $k_{d 2}$, correspond to Hopf bifurcation points. Based on the method of multiple scales, we obtained the normal form of the Hopf bifurcation. By numerically evaluating the coefficients of the normal form for several sets of parameters, we showed that the each of the Hopf bifurcations can be supercritical or subcritical. For the parameter values we considered, both bifurcations were subcritical for small enough delay. We considered one set of parameter values in more detail. For this set, the Hopf bifurcation at $k_{d 1}$ is subcritical and that at $k_{d 2}$ is supercritical. This means that an unstable limit cycle coexists with the stable equilibrium point for $k_{d}>k_{d 1}$ and at least close to $k_{d 1}$. Numerical simulation and numerical continuation studies of the full model confirm the predictions of the analysis, and indicate that the unstable limit cycle exists for $k_{d} \in\left(k_{d 1}, k_{d 2}\right)$. We saw similar behaviour in numerical studies where the position feedback gain, $k_{p}$, or the acceleration feedback gain, $k_{a}$, was used as the continuation parameter. This means that large enough perturbations may cause instability in the system even if the feedback is chosen to stabilize the equilibrium position. This nonlinear instability mechanism due to a subcritical Hopf bifurcation has been observed in other systems with delay [28, 29, $30,35,36,37]$. The fact that subcritical Hopf bifurcations exist for all the parameter values we tested means that this phenomenon may be quite prevalent in the maglev system. Thus linear control theory/stability analysis may not be adequate to guarantee good performance of the system.

Vibration phenomena are profuse when the maglev vehicle runs on the guideway. To 
completely understand the dynamic behavior of the maglev system, further research needs be carried out. Numerical bifurcation analysis can determine the amplitude of unstable limit cycles which co-exist with the stable equilibrium state. This would help quantify how robust the stability is to perturbations. A more extensive exploration of parameter space may yield values of the feedback gains or physical parameters which give better performance of the system. Our analysis has shown that the system can have codimension 2 Hopf-Hopf bifurcation points. In the particular example we considered the frequencies of the Hopf bifurcations were in 1:2 resonance and a secondary period doubling bifurcation occurred. If the Hopf bifurcations are not resonant, then quasiperiodicity or multistability between different periodic solutions can occur. The resulting complex dynamics would likely degrade the performance of the maglev system. Thus a more detailed study of when such points occur and the behaviour associated with them would be useful. Finally, in some of our numerical studies the instability was large enough that the train would come in contact with the rail, at which point our model becomes invalid. It would be interesting to extend the model to include the effect of impacts with the rail. The resulting hybrid model would presumably be related to impact oscillator models, which have been shown to exhibit a variety of interesting behaviour such as grazing bifurcations [38]. The study of machine tool dynamics can lead to models with both impacts and time delay. Chaotic behaviour has been shown to arise in such models [39, 40].

\section{Acknowledgements}

This work was supported by 973 Program (2009CB326202), National Natural Science Foundation of China (11071060,60835004), Key Program of Hunan Basic Research for Applications (2008F J2008), Aid program for Science and Technology Innovative Research Team in Higher Educational Institutions of Hunan Province (XJT2008-244) and the Discovery Grants Program, Natural Sciences and Engineering Research Council of Canada. Zhizhou Zhang (National University of Defense Technology, Hunan) provided helpful discussions of the model and how it relates to experimental maglev systems. 


\section{References}

[1] Y. Yoshihide, F. Masaaki, T. Masao, The First HSST Maglev Commercial Train in Japan, Maglev 2004 Proceedings 1 (2004) 76-85.

[2] G. Roger, Dynamic and Control Requirements for EMS Maglev Suspension, Maglev 2004 Proceedings 2 (2004) 926-934.

[3] Z. Zhang, Z. Long, L. She, W. Chang, Linear Quadratic State Feedback Optimal Control against Actuator Failures, Proceedings of the 2007 IEEE International Conference on Mechatronics and Automation (2007) 3349-3354.

[4] M. Attilio, Vibration control for parametrically exicited Lineard systems, Int. J. Nonlinear Mech. 41 (2006) 146-155.

[5] M. Attilio, Vibration control for primary resonance of the van der Pol oscillator by a time-delay state feedback, Int. J. Non-linear Mech. 38 (2003) 123-131.

[6] M. Attilio, Vibration control for the primary resonance of a cantilever by time-delay state feedback, J. Sound and Vibration 259 (2003) 241-251.

[7] E. Stone, S. Campbell, Stability and bifurcation analysis of a nonlinear DDE model for drilling, J. Nonlinear Sci. 14(1) (2004) 27-57.

[8] G. Stépán, G. Haller, Quasiperiodic oscillations in robot dynamics, Nonlinear Dyn. 8 (1995) 513-528.

[9] G. Orosz, G. Stépán, Hopf bifurcation calculations in delayed systems with translational symmetry, Journal of Nonlinear Sci. 14(6) (2004) 505-528.

[10] N.A. Nayfeh, W.T. Baumann, Nonlinear analysis of time-delay position feedback control of container cranes, Nonlinear Dyn. 53 (2008) 75-88.

[11] H.P. Wang, J. Li, K. Zhang, Stability and Hopf bifurcation of the maglev system with delayed speed feedback control, Acta Automatica Sinica 33(8) (2007) 829-834. 
[12] L.L. Zhang, L.H. Huang, Z.Z. Zhang, Stability and Hopf bifurcation of the maglev system with delayed position and speed feedback control, Nonlinear Dyn. (2009) 57(12) $197-207$.

[13] L.L. Zhang, L.H. Huang, Z.Z. Zhang, Hopf bifurcation of the maglev time-delay feedback system via pseudo-oscillator analysis, Math. Comp. Model. (2010) 52 667-673.

[14] A.H. Nayfeh, Perturbation Methods. Shanghai Publishing House of Science and Technology, Shanghai (1984).

[15] A.H. Nayfeh, Method of Normal Forms, Wiley Interscience, New York (1993).

[16] A.H. Nayfeh, C.M. Chin, J.R. Pratt, Perturbation methods in nonlinear dynamics: applications to machining dynamics, J. Manuf. Sci. Technol. 119 (1996) 485-493.

[17] Y.G. Zheng, Z.H. Wang, Stability and Hopf bifurcation of an optoelectronic time-delay feedback system, Nonlinear Dyn. 57 (2009) 125-134.

[18] Z.N. Masoud, A.H. Nayfeh, A. Al-Mousa, Delayed position-feedback controller for the reduction of payload pendulations of rotary cranes, J. Vib. Control 9(1) (2003) 257-277.

[19] A.H. Nayfeh, Order reduction of retarded nonlinear systems - the method of multiple scales versus center-manifold reduction. Nonlinear Dyn. 51(4) (2008) 483-500.

[20] Z. Zhang, Z. Long, L. She and W. Chang, Fault-tolerant Control for Maglev Suspension System Based on Simultaneous Stabilization, Proceedings of the IEEE International Conference on Automation and Logistics (2007) 299-303.

[21] J.F. Whidborne, EMS control system design for a maglev vehicle - A critical system, Automatica 29(5) (1993) 1345-1349.

[22] J.K. Hale and S.M. Verduyn Lunel, Introduction to Functional Differential Equations, Springer Verlag, New York 1993.

[23] F. Takens, Unfoldings of certain singularities of vector fields: generalized Hopf bifurcations. JDE 14 (1973) 476-493. 
[24] M. Golubitsky and W.F. Langford, Classification and unfoldings of degenerate Hopf bifurcation. JDE 41 (1981) 525-546.

[25] K. Engelborghs, T. Luzyanina, and G. Samaey, DDE-BIFTOOL v. 2.00: a Matlab package for bifurcation analysis of delay differential equations. Technical Report TW330, Department of Computer Science, K.U. Leuven, Leuven, Belgium (2001).

[26] V.G. LeBlanc and W.F. Langford, Classification and unfoldings of $1: 2$ resonant Hopf bifurcation. Arch. Rational Mech. Anal. 136 (1996) 305-357.

[27] S.A. Campbell and V.G. LeBlanc. Resonant Hopf-Hopf interactions in delay differential equations. JDDE 10(2) (1998) 327-346.

[28] G. Stépán and T. Kalmár-Nagy. Nonlinear Regenerative machine tool vibrations. Proceedings of DETC'97. Sacramento, California.

[29] T. Kalmár-Nagy, G. Stépán and F.C. Moon. Subcritical Hopf bifurcation in the delay equation model for machine tool vibrations. Nonlinear Dyn. 26 (2001) 121-142.

[30] L. Larger, J.-P. Goedgebuer and T. Erneux. Subcritical Hopf bifurcation in dynamical systems described by a scalar nonlinear delay differential equation. PRE 69 (2004) 036210 .

[31] G. Stépán, Retarded Dynamical Systems: Stability and Characteristic Functions, Pitman Research Notes in Mathematics Series, Vol. 210, Wiley \& Sons, New York (1989).

[32] F. Atay, Balancing the inverted pendulum using position feedback, Appl. Math. Lett. 12 (1999) 51-56.

[33] L. Kollár, G. Stépán, and S. Hogan, Sampling delay and backlash in balancing systems, Periodica Polytechnica Ser. Mech. Eng., 44 (2000) 77-84.

[34] J. Milton et al. The time-delayed inverted pendulum: Implications for human balance control, CHAOS 19 (2009) 026110. 
[35] G. Orosz and G. Stépán. Subcritical Hopf bifurcations in a car-following model with reaction-time delay. Proc. Royal Soc. A 462 (2006) 2643-2670.

[36] S.A. Campbell and E. Stone, Analysis of the chatter instability in a nonlinear model for drilling. ASME J. Comp. Nonl. Dyn. 1 (2006) 294-306.

[37] S.A. Campbell, S. Crawford and K. Morris, Friction and the inverted stabilization problem, ASME J. Dyn. Sys. Meas. Cont., 130(5) (2008) 054502-054508.

[38] W. Chin et al., Grazing bifurcations in impact oscillators, Phys. Rev. E. 50(6) (1994) 4427-4444.

[39] G. Csernák and Zoltán Pálmai, Exploration of the chaotic phenomena induced by fast plastic deformation of metals, Int. J. Adv. Manuf. Tech. 40 (2009) 270-276.

[40] Z. Dombovari et al., On the global dynamics of chatter in the orthogonal cutting model, Int. J. Non-linear Mech. 46 (2011) 330-338. 